\title{
Factores asociados a la resiliencia y adherencia terapéutica en pacientes con injerto renal funcionante
}

\author{
Yolanda Doalto Muñoz, Raquel Cruz Valle, María Luisa Carretón Manrique \\ Enfermeras. Hospital Universitario Marqués de Valdecilla. Cantabria. España
}

\section{Resumen}

Introducción: El aumento en el número de trasplantes de órganos y la escasa adherencia a los tratamientos una vez realizado el trasplante suscita una actitud de alerta entre los profesionales de enfermería y un problema de salud pública.

Objetivo: Conocer los factores asociados a la resiliencia que influyen sobre la adherencia al tratamiento, en este caso en el trasplante con injerto renal.

Material y Método: Estudio descriptivo trasversal y retrospectivo en población adulta trasplantada con injerto funcionante desde enero de 2012 hasta junio de 2016 con edades comprendidas entre 18 y 70 años, de ambos sexos, autosuficientes en el manejo de tratamiento; y trasplantados en el Hospital Marqués de Valdecilla. Pertenecientes, como socios, a la Asociación Alcer Cantabria.

Resultados: Los resultados obtenidos, aun no siendo significativos por el tamaño muestral, demuestran una influencia positiva ( $3 \%$ de la variabilidad) de la resiliencia en la adherencia al tratamiento. Se demuestran que existen diferencias significativas en alguna de las variables sociodemográficas como la actividad profesional, nivel de estudios y edad. Así mismo, se encuentran diferencias según el nivel de tolerancia a los efectos negativos y estrés.

Conclusiones: Los datos revelan mayor riesgo de no adherencia en el grupo de menor edad, por lo que resulta necesario sensibilizar de la importancia de la adherencia

\begin{tabular}{|c|}
\hline Correspondencia: \\
Yolanda Doalto Muñoz \\
Servicio de Traumatología y Cirugía Plástica \\
Planta 7, Torre B. Hospital Universitario Marqués de Valdecilla \\
39008 Santander \\
E-mail: litha_10@hotmail.com
\end{tabular}

al tratamiento. Sorprende la relación inversa entre baja adherencia en pacientes y mayor control situacional.

PALABRAS CLAVE: resiliencia psicológica; trasplante de riñón; cumplimiento; adherencia al tratamiento.

\section{Factors associated with resilience and therapeutic adherence in patients with functioning renal graft}

\section{Abstract}

Introduction: The increase in the number of organ transplants and the poor adherence to the treatments after transplant provokes an alert attitude among the nursing professionals and a public health problem.

Aim: To know the factors associated with resilience, which influence adherence to treatment, in this case in kidney graft transplantation.

Material and Method: A cross-sectional and retrospective descriptive study was carried out in adult population transplanted with functioning graft, from January 2012 to June 2016 with ages between 18 and 70 years, of both sexes, self-sufficient in the treatment management; and transplanted in the Marqués de Valdecilla Hospital. Belonging, as partners, to the Alcer Cantabria Association.

Results: The results obtained, although not being significant due to the sample size, demonstrate a positive influence ( $3 \%$ of the variability) of the resilience in the adherence to the treatment. It is shown that there are significant differences in some of the sociodemographic variables such as professional activity, educational level and age. Likewise, there are differences according to the level of tolerance to negative effects and stress. 
Conclusions: The data reveal a greater risk of nonadherence in the younger age group, so it is necessary to raise awareness of the importance of adherence to treatment. The inverse relationship between low adherence in patients and greater situational control is surprising.

KEYWORDS: psychological resilience; kidney transplant; compliance; treatment adherence

\section{Introducción}

El trasplante de órganos se presenta como una alternativa terapéutica para aquellos pacientes con graves problemas en el funcionamiento de un órgano vital ${ }^{1}$. El trasplante renal es en la actualidad y desde hace años la terapia de elección para la mayoría de las causas de insuficiencia renal en la que pueda estar indicado? Estos problemas, suponen un conjunto de condicionantes en su estilo de vida, tales como: restricciones en la dieta, en las actividades diarias, disfunciones sexuales y en el caso del riñón dependencia de la diálisis ${ }^{1,2}$.

El trasplante de órganos ofrece a los pacientes mejor calidad de vida y, en principio, más años de vida. No obstante, y en ningún caso, este trasplante, es una solución total a su patología, puesto que siempre está latente el riesgo del rechazo. Esta permanente situación puede generar problemas psicológicos tanto el paciente como a su familia, durante el proceso y a lo largo de la vida.

Especialmente la fase o proceso post-trasplante, es un periodo de conflictos personales y familiares ambivalentes que oscilan entre el miedo y la esperanza, la dependencia y la independencia ${ }^{1}$. En este proceso, resulta de especial trascendencia una buena adherencia terapéutica para la supervivencia del órgano trasplantado. Sin embargo, y a pesar de su importancia muchos pacientes no desarrollan una buena adherencia terapéutica.

Según algunos estudios, es difícil detectar precozmente el incumplimiento, aunque se han descrito perfiles con posible mal seguimiento se puede dar también en pacientes que a priori parecen bien adaptados ${ }^{3}$.

En la actualidad, el porcentaje estimado de pacientes trasplantados que muestran falta de cumplimiento terapéutico se sitúa entre el $20 \%$ y el $54 \%$. En el trasplante renal, este incumplimiento, se estima que contri- buyó al $20 \%$ de rechazos agudos y al $16 \%$ de pérdida de injertos. Lo cual supone un importante problema de salud pública ${ }^{4}$.

Se entiende por adherencia terapéutica, al grado de coincidencia entre el comportamiento de un paciente en relación con los medicamentos que hay que tomar, el seguimiento de la dieta o los cambios que ha de hacer en su estilo de vida, y las recomendaciones de los profesionales de salud que les atienden. Para que la adherencia se lleve a cabo, el paciente debe tener un estilo de vida físico, emocional, social y laboral favorable a su nueva situación de paciente trasplantado 5 .

En los últimos años, los organismos internacionales de salud, como la Organización Mundial de salud y la Organización Panamericana de Salud, en sus programas división, protección y provisión de salud ${ }^{6}$, han sugerido usar el enfoque de la resiliencia, entendida como: "la capacidad humana para enfrentarse, sobreponerse y ser fortalecido o transformado por experiencias de adversidad". En base a esta definición podemos decir que se refiere a la capacidad de la persona para mantener un funcionamiento efectivo frente a las adversidades del entorno o para recuperarlo en otras condiciones ${ }^{7}$.

Kobasa y cols $^{8}$ mencionaron por primera vez el concepto de "personalidad resiliente" en 1982, en relación con la idea de protección frente a los factores que provocan estrés. Es así que establecieron que las personas con características resilientes presentan un gran sentido del compromiso, una fuerte sensación de control sobre los acontecimientos, están más abiertos a los cambios, a la vez que tienden a interpretar las experiencias estresantes y adversas como una parte más de la vida. Estos autores consideran la "personalidad resiliente" como un constructo multifactorial con tres componentes principales: compromiso, control y reto. La investigación basada en la evidencia ha sido concluyente al afirmar que la resiliencia es una variable protectora de salud física y mental en momentos de enfermedad. Una alta resiliencia se puede asociar a una buena adherencia en el tratamiento.

Entre los estudios realizados sobre este tema se encuentra el de Farber y cols $^{9}$ quienes investigaron los factores de resiliencia asociados a la adaptación a la enfermedad del VIH/SIDA y encontraron que altos niveles de resiliencia estaban asociados con bajo distrés, mejor calidad de vida y creencias personales positivas, mejor sentido y propósito por la vida y mejor adapta- 
ción a la enfermedad. Es importante resaltar que la resiliencia puede ser fomentada, es decir, es una capacidad universal pero no es absoluta ni estable, por lo que una persona puede ser resiliente en un medio y no en otro ${ }^{7}$. Así, la teoría de la resiliencia cobra significado a partir de las diferencias en la reacción ante circunstancias adversas, generadoras de estrés ${ }^{7}$. Mientras algunas personas sucumben ante dichas situaciones, evidenciando desequilibrio y trastornos a diversos niveles, otras fomentan un proceso de adaptación exitosa y transformación a pesar de la adversidad.

Nos planteamos como objetivo en esta investigación, determinar si existe relación significativa entre el nivel de resiliencia, la adherencia al tratamiento y sus factores asociados, en una población de trasplantados renales.

\section{Material y Método}

Estudio descriptivo transversal prospectivo, en pacientes trasplantados renales desde enero de 2012 hasta junio de 2016, con injerto funcionante en la actualidad. Se incluyeron en el estudio sujetos mayores de 18 años, autónomos en su autocuidado, y pertenecientes, como socios, a la Asociación ALCER Cantabria.

Se realizó un muestreo intencional, no probabilístico. El reclutamiento de sujetos fue realizado por la trabajadora social de ALCER en la Asociación, informando sobre la relevancia del estudio y solicitando el consentimiento informado.

Para determinar el nivel de resilencia se utilizó la escala de resiliencia de Connor-Davidson (CD-RISC) en su versión validada en población española ${ }^{9,10}$. Esta escala consta de 25 ítems estructurados en una escala sumativa tipo Likert, que en su versión original se agrupa en cinco dimensiones:

1. Competencia personal, metas altas y tenacidad.

2. Tolerancia a los efectos negativos y hacer frente a los efectos del estrés.

3. Efectos positivos del cambio y la seguridad en las relaciones.

4. Control.

5. Influencia espiritual.

La persona ha de indicar hasta qué punto cada una de las afirmaciones ha sido verdadera en su caso durante el último mes en una escala de $0-4$, donde $0=$ "no ha sido verdadera en absoluto" y $4=$ "verdadera casi siempre". Las puntuaciones totales oscilan entre $0 \mathrm{y}$ 100; a mayores puntuaciones, mayor resiliencia (no tiene establecido un punto de corte $)^{10}$.

Para determinar la adherencia, se utilizó la Escala Informativa de Cumplimiento de la Medicación (The Medication Adherence Report Scale, MARS)11,12. Consta de 30 preguntas que se agrupan en tres dimensiones sobre:

\section{Creencias \\ 2. Experiencias \\ 3. Comportamiento en salud.}

Permite identificar los predictores de cumplimiento de la medicación en pacientes con enfermedades crónicas y contrastar el buen cumplimiento autorreferido. Los encuestados indican su grado de acuerdo o desacuerdo con afirmaciones sobre el uso de su medicamento en una escala Likert de 5 puntos. Una puntuación de 25 indica buen cumplimiento, mientras que si es menor de 25 revela un cumplimiento subóptimo. En nuestro caso, la puntuación en la que se estimó como adherente o no, se estipuló en 19 puntos.

Así mismo se recogieron otras variables sociodemográficas: Edad; Sexo (mujer/hombre); Nivel académico (Básicos, formación profesional, universitarios); Actividad laboral (activos en los que se incluyen los parados; jubilados); Estado civil (soltero; casado; viudo).

La distribución y recogida de las escalas en un plazo de 15 días tras la entrega del material, compuesto por: el documento de consentimiento informado y ambas escalas de medida (CD-RISC y MARS) se llevó a cabo por la trabajadora social de ALCER.

Con carácter previo, se pilotó la cumplimentación de las escalas y se enviaron a 5 socios quienes las rellenaron y entregaron correctamente en un plazo inferior a 15 días.

El abordaje estadístico se llevó a cabo con el programa IBM SPSS 25. Las variables y sus subescalas se presentaron con la mediana, mientras que la representación gráfica se realizó a través de diagramas de dispersión y diagramas de cajas. Se concretó el análisis de significación estadística para $p=0,05$, considerándose valores inferiores, estadísticamente significativos. La relación entre las variables se cotejó con las pruebas no paramétricas Mann-Whitney y Kruskal-Wallis. Aten- 
diendo a este criterio de normalidad, se utilizó el test no paramétrico de Rho de Spearman para el análisis correlacional.

El proyecto se desarrolló siguiendo los principios éticos recogidos en la declaración de Helsinki para las investigaciones médicas en seres humanos; y la Ley Orgánica 15/1999, de 13 de diciembre, de protección de datos de carácter personal. El proyecto fue aprobado por del Comité Ético de Investigación Clínica de Cantabria.

\section{Resultados}

Se incluyeron en el estudio un total de 18 personas con trasplante renal funcionante. En cuanto a las características sociodemográficas, que se exponen dentro de la Tabla 1, destaca el predominio de varones, con una edad inferior de 50 años, un tercio tenían estudios universitarios, la mayoría estaban casados y la mitad jubilados.

En la Tabla 2 se muestran las puntuaciones promedio (medianas) en las escalas de adherencia y resiliencia. En cuanto a la resiliencia se aprecia que las mujeres, los pacientes con edad $\geq 50$ años y los que poseen estudios universitarios, son los que obtienen puntuaciones mayores de resiliencia, aunque ninguna de las diferencias alcanzó el nivel de significación. Los pacientes que mostraron unas puntuaciones mayores en el P75 de adherencia también fueron los de las categorías mencionadas anteriormente para resiliencia, es decir: mujeres, $\geq 50$ años y con estudios universitarios; aunque tampoco fueron significativas las diferencias encontradas.

En la Tabla 3 se aprecia que los pacientes considerados como adherentes tienen puntuaciones más altas en la dimensión "tolerancia a efectos negativos"; además también se constata como los pacientes considerados como

Tabla 2. Puntuaciones en los cuestionarios de adherencia y resiliencia para el total de la muestra y según las variables sociodemográficas: edad, sexo, nivel de estudios y actividad profesional. P25: percentil 25. P75: percentil 75.

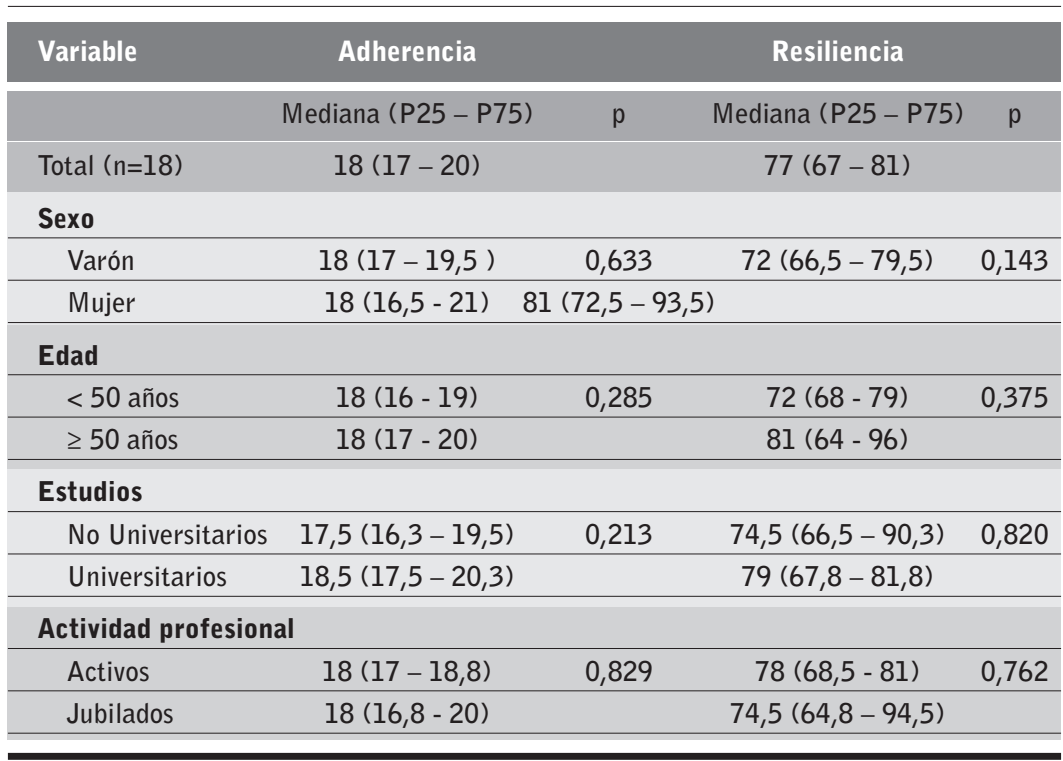

Tabla 1. Características sociodemográficas de los pacientes incluidos en el estudio. FP: Formación profesional.

\begin{tabular}{|c|c|c|}
\hline Variable & $\mathbf{N}$ & $\%$ \\
\hline \multicolumn{3}{|l|}{ Sexo } \\
\hline Varón & 13 & 72 \\
\hline Mujer & 5 & 28 \\
\hline \multicolumn{3}{|l|}{ Edad } \\
\hline$<50$ años & 11 & 61 \\
\hline$\geq 50$ años & 7 & 39 \\
\hline \multicolumn{3}{|l|}{ Estudios } \\
\hline Básicos & 5 & 28 \\
\hline $\mathrm{FP}$ & 7 & 39 \\
\hline Universitarios & 6 & 33 \\
\hline \multicolumn{3}{|l|}{ Estado civil } \\
\hline Casado/a & 13 & 73 \\
\hline Soltero/a & 4 & 22 \\
\hline Viuda & 1 & 6 \\
\hline \multicolumn{3}{|c|}{ Actividad profesional } \\
\hline Ama de casa & 2 & 11 \\
\hline Desempleado/a & 2 & 11 \\
\hline Activo/a & 5 & 28 \\
\hline Jubilado & 9 & 50 \\
\hline
\end{tabular}

no adherentes tienen puntuaciones más altas en la dimensión "control", no obstante, en ninguno de los dos casos las diferencias fueron significativas.

La figura 1 muestra los resultados de medida comparativa de las dos variables, Adherencia y Resiliencia. 
Tabla 3. Comparación de las puntuaciones en las cinco dimensiones de la escala de resiliencia entre pacientes adherentes versus no adherentes.

\begin{tabular}{lccc}
\hline Variable & Adherencia $(\mathbf{n = 6})$ & No Adherencia $(\mathrm{n}=12)$ & \\
& Mediana $(\mathrm{P} 25-\mathrm{P} 75)$ & Mediana $(\mathrm{P} 25-\mathrm{P} 75)$ & $\mathrm{p}$ \\
\hline Competencia personal & $25,5(22,4-31,4,0)$ & $25,5(21,2-28,7)$ & 0,574 \\
\hline Tolerancia efectos negativos & $25,0(21,0-27,0)$ & $20,0(18,5-25,0)$ & 0,242 \\
\hline Efectos positivos & $15,5(15,0-16,0)$ & $16,0(13,5-18,0)$ & 0,851 \\
\hline Control & $8,5(8,0-10,0)$ & $10,5(8,5-11,5)$ & 0,301 \\
\hline Influencia espiritual & $4,0(1,0-5,0)$ & $4,0(3,5-7,0)$ & 0,538 \\
\hline Total Resiliencia & $77,0(69,0-81,0)$ & $75,0(67,0-82,5)$ & 0,963 \\
\hline \hline
\end{tabular}

ciona más directamente con la no adherencia. En nuestro estudio, se identifica el grupo de menor edad como el de mayor riesgo de pérdida de injerto renal por su menor adherencia y baja resiliencia.

No ocurre lo mismo en cuanto a los resultados de medida del nivel académico puesto que en algunos estudios considera que no es una variable influyente, por el contrario,

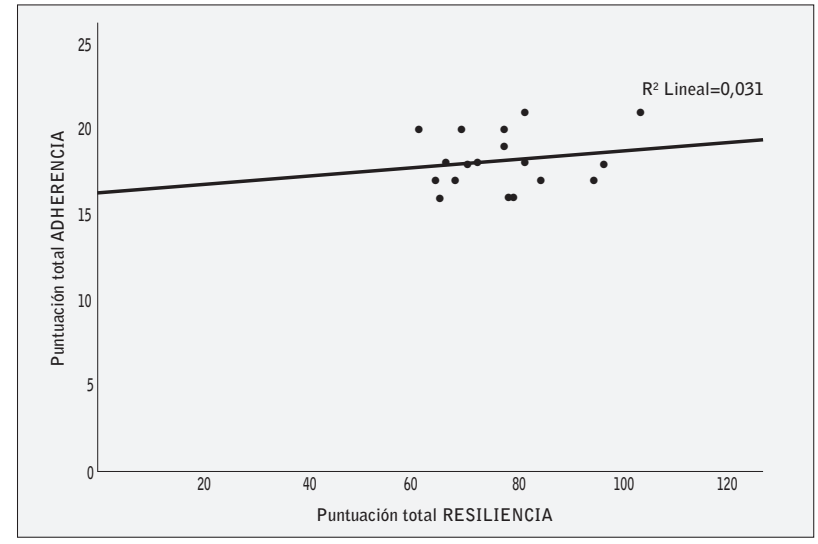

Figura 1. Relación entre las puntuaciones totales obtenidas en la escala de resiliencia y adherencia.

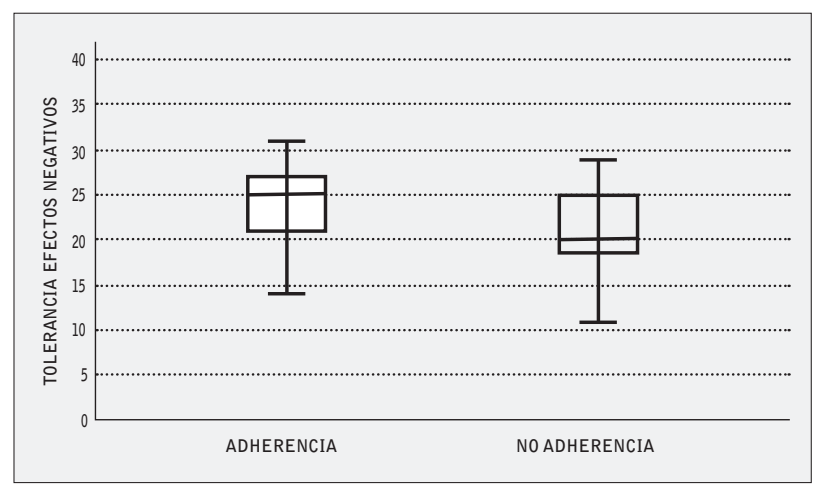

Figura 2. Puntuaciones en la dimensión de "tolerancia a efectos negativos" entre pacientes con y sin adherencia.

\section{Discusión/Conclusiones}

Algunas de las características demográficas del grupo de estudio se comparten con otros trabajos ${ }^{13}$. Se demuestra que existen diferencias significativas en alguna de las variables, como la actividad profesional, nivel de estudios y edad, siendo esta última, la que se rela- nuestros resultados demuestran una discreta tendencia a mayor resilencia cuanto mayor nivel de estudios ${ }^{13}$.

En cuanto a las dimensiones de la resiliencia que se mostraron más relacionadas con la adherencia, creemos que son la "tolerancia a efectos negativos" y el "control". Ambas pueden tener utilidad en la práctica clínica, dado que, si se identifica a los pacientes con menor tolerancia a los efectos negativos que serán los previsiblemente menos adherentes al tratamiento inmunosupresor, se puede establecer una mayor vigilancia para detectar precozmente el posible incumplimiento del tratamiento prescrito, esto permitirá una mayor supervivencia del trasplante. Por otro lado, los pacientes identificados con una mayor autopercepción de "control" son los más proclives a tomar, por su propia iniciativa, la decisión de abandonar el tratamiento pautado, con el consiguiente riesgo de rechazo del trasplante.

En relación con la resiliencia, otros autores refieren que juega un papel importante como variable protectora en la calidad de vida a nivel de salud mental de los pacientes, relacionando que niveles altos de resiliencia se asocian con menor riesgo de sufrimiento psicológi$\mathrm{Co}^{14,15}$. Se sugiere que la resiliencia individual puede ser modificada, pudiendo tener un efecto positivo sobre la adherencia ${ }^{14}$.

Por nuestra parte, se propone un programa de apoyo al inicio del proceso de trasplante para fortalecer la resiliencia y así mejorar el proceso de rehabilitación física y emocional, como el que se desarrolla desde la Asociación Psicológica Americana, donde se promueve un programa de 10 formas de construir la resiliencia, trabajando como establecer relaciones, evitar considerar las crisis como problemas insalvables, aceptar que los cambios son partes de la vida. 
Debido al limitado número muestral, los resultados y conclusiones del estudio deben ser tomados con cautela. En este sentido, el estudio debe ser considerado exploratorio, con la finalidad de sugerir posibles variables que pueden explicar la falta de adherencia al tratamiento en los pacientes trasplantados, que deberán ser evaluadas con futuros estudios.

Sería preciso realizar el estudio aumentando el tamaño muestral y continuar en esta línea de investigación sobre la importante consecuencia de la no adherencia en la pérdida del injerto de los pacientes trasplantados.

\section{Agradecimientos}

Agradecemos la colaboración de Da Laura Marcos Diaz, trabajadora social de la asociación ALCER Cantabria.

\section{El autor declara que no hay conflicto de interés.}

Recibido: 27 abril 2018

Revisado: 5 mayo 2018

Modificado: 20 mayo 2018

Aceptado: 25 mayo 2018

\section{Bibliografía}

1. Pérez San Gregorio MA, Rodríguez AM, Rodríguez AG. Problemas psicológicos asociados al trasplante de órganos. Int $\mathrm{J}$ Clin Health Psychol 2005;5(1):99-114.

2. Martín P, Errasti P. Trasplante renal. An. Sist. Sanit. Navar. 2006;29(Supl 2):S79-92.

3. Barquero Ruano RM, Checa Barambio E, Rodríguez Peña J. Mala adherencia al tratamiento inmunosupresor de un paciente trasplantado renal. Rev Soc Esp Enferm Nefrol 2010;13(4):267-269.

4. Ortega Suarez FJ, Sánchez Plumed J, Pérez Valentín MA, Pereira Palomo P, Muñoz Cepeda MA,

Lorenzo Aguiar D. Validación del cuestionario simplificado de adherencia a la medicación (SMAQ) en pacientes con trasplante renal en terapia con tacrolimus. Nefrologia 2011;31(6):690-6.

5. Ruiz Flores F. Adherencia al tratamiento farmacológico de los pacientes con hipertensión arterial sistémica en una unidad de medicina familiar [Tesis]. Facultad de enfermería. Universidad Cesar Vallejo, ciudad de Lima; 2015.

6. Munist $M$, Santos $H$, Kotliarenco $M A$, Suárez Ojeda EN, Infante F, Grotberg E. Manual de identificación y promoción de la resiliencia en niños y adolescentes. Washington: Organización Panamericana de la Salud, Organización Mundial de la Salud, la Fundación WK Kellogg y la Autoridad Sueca para el Desarrollo Internacional (ASDI); 1998.

7. Bedón López YL. Resiliencia y adherencia al TARGA en pacientes con VIH/SIDA del Hospital San Juan Bautista de Huaral. Psique Mag 2013;2 (1):51-63.

8. Acosta López de Llergo IC, Sánchez Araiza YI. Manifestación de la Resiliencia como Factor de Protección en Enfermos Crónico Terminales Hospitalizados. Psicol. iberoam. 2009;17(2):24-32.

9. Quiceno JM, Vinaccia S. Resiliencia: una perspectiva desde la enfermedad crónica en población adulta. Pensam. psicol. 2011;9(17):69-82.

10. Crespo M, Fernández-Lansac V, Soberón C. Adaptación española de la "Escala de resiliencia de Connor-Davidson" (CD-RISC) en situaciones de estrés crónico. Psicol Conduct 2014;22(2):21938.

11. Thompson K, Kulkarni J, Sergejew AA. Reliability and validity of a new Medication Adherence Rating Scale (MARS) for the psychoses. Schizophr Res. 2000;42:241-247.

12. Johnson, $G$ et al. Factors associated with medication nonadherence in patients with COPD. Chest, 2005;128(5):3198-3204.

13. Ruiz Calzado MR. La adherencia terapéutica en el paciente trasplantado renal. Enferm Nefrol 2013;16(4):258-70. 
14. Vinaccia S, Quiceno JM. Resiliencia y calidad de vida relacionada con la salud en pacientes con insuficiencia renal crónica-IRC. Rev Argent Clín Psicol 2011;20(3):201-11.

15. Tian X, Gao Q, Li G, Zou G, Liu C, Kong L, Li P. Resilience is associated with low psychological distress in renal transplant recipients. Gen Hosp Psychiatry. 2016;39:86-90.

Este artículo se distribuye bajo una Licencia Creative Commons Atribución-NoComercial 4.0 Internacional. https://creativecommons.org/licenses/by-nc/4.0/

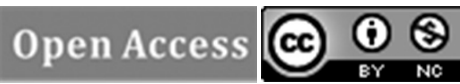

